Relations industrielles

Industrial Relations

\title{
Loi des relations ouvrières
}

\section{Marius Bergeron}

Volume 2, numéro 3, novembre 1946

URI : https://id.erudit.org/iderudit/1023826ar

DOI : https://doi.org/10.7202/1023826ar

Aller au sommaire du numéro

Éditeur(s)

Département des relations industrielles de l’Université Laval

ISSN

0034-379X (imprimé)

1703-8138 (numérique)

Découvrir la revue

Citer cet article

Bergeron, M. (1946). Loi des relations ouvrières. Relations industrielles /

Industrial Relations, 2(3), 3-6. https://doi.org/10.7202/1023826ar

Tous droits réservés (C Département des relations industrielles de l’Université Laval, 1946
Ce document est protégé par la loi sur le droit d'auteur. L'utilisation des services d'Érudit (y compris la reproduction) est assujettie à sa politique d'utilisation que vous pouvez consulter en ligne.

https://apropos.erudit.org/fr/usagers/politique-dutilisation/ 


\section{LOI DES RELATIONS OUVRIĒRES}

NDLR. - Nous présentons ici sous une forme plus claire la Loi des relations ouvrières (S.R.Q., 1941, chapitre 162 A) telle qu'amendée et du Règlement numéro 1 de la Commission de relations ouvrières. De cette façon, nous croyons que nos lecteurs pourront en avoir une vue d'ensemble complète et en faire plus facilement l'étude.

\section{NOTES PRELIMINAIRES}

I. - Quels sont les principes consacrés par la loi?

1) le principe de la liberté d'association ;

2) le principe des négociations obligatoires.

II. - Généralités :

1) l'objet de la loi est de déterminer le ou les agents pour fins de négociations ;

2) quiconque, pour les fins de la loi, peut se faire représenter par des représentants dûment mandatés ;

3) tout document exigé ou produit à la Commission doit lui être transmis en deux copies, à son siège social, en la cité de Québec, numéro 286, rue Saint-Joseph.

\section{CHAPITRE PREMIER}

\section{ETUDE DES PRINCIPES CONSACRES} PAR LA LOI

Section A

\section{La liberté d'association}

\section{III. - Qui jouit du droit d'association?}

Tout employeur et tout employé.

Explication: dans le texte de la loi, nous lisons le mot " salarié ", au lieu du mot " employé ».

\section{IV. - Comment la loi protège-t-elle le droit d'asso- ciation?}

Par des pratiques interdites.

\section{V. - A quelles personnes s'appliquent ces prati- ques interdites?}

1) Aux employeurs : c'est-à-dire à eux-mêmes, à leurs agents et à leur association, interdiction de :

a) dominer ou entraver la formation ou les activités d'un syndicat ;

Explication : dans le texte de la loi, nous lisons le mot " association ", au lieu du mot " syndicat".

b) refuser d'employer une per- l'employeur peut cesonne parce qu'elle est membre pendant suspendre, ou officier d'un syndicat ; démettre, renvoyer

c) contraindre de quelque fa- ou déplacer un emçon que ce soit un employé de ployé pour cause juss'abstenir ou de cesser d'être te et raisonnable dont membre d'un syndicat; la preuvelui incombe.

d) d'ordonner, d'encourager ou de supporter une contre-grève, c'est-à-dire, un refus de fournir du travail à un groupe d'employés ;

e) de ne pas reconnaitre un syndicat qui détient un certificat de reconnaissance.

2) Aux agents des employés : interdiction de

a) solliciter pendant les heures de travail des adhésions à un syndicat ;

b) réunir au lieu du travail des em- sans le conployés dans le but de solliciter leur adhé- l'employeur. sion à un syndicat;

3) Aux agents des employés et aux syndicats : interdiction de

a) adhérer à une association d'employeurs ;

b) dominer ou entraver la formation ou les activités d'une association d'employeurs ;

c) d'ordonner, d'encourager ou de supporter une grève, c'est-à-dire une cessation concertée de travail par un groupe d'employés.

4) A tous : interdiction

d'user $\left\{\begin{array}{l}\text { d'intimidation; } \\ \text { de menaces, }\end{array}\right.$
pour amener quiconque à $\left\{\begin{array}{l}\begin{array}{l}\text { devenir mem- } \\ \text { bre, } \\ \text { s'abstenir de } \\ \text { devenir mem- } \\ \text { bre, } \\ \text { cesser d'être } \\ \text { membre. }\end{array}\end{array}\right\} \begin{aligned} & \text { d'un syndicat } \\ & \text { ou } \\ & \text { d'une associa- } \\ & \text { tion d'employ- } \\ & \text { eurs. }\end{aligned}$

\section{VI. - La loi limite-t-elle ce droit d'association?}

Oui, car sauf dans les soixante (60) jours précédant la date d'expiration ou de renouvellement de la convention,

1) une association d'employeurs ou un syndicat qui a conclu une convention collective ;

2) un groupe d'employeurs ou d'employés, membres d'une association d'employeurs ou d'un syndicat. ne feront des démarches en vue de s'affilier à une autre association d'employeurs ou à un autre syndicat; devenir membre d'une autre association : d'employeurs ou d'un autre syndicat.

Section B

\section{Les négociations obligatoires}

\section{VII. - Quelles sont les personnes que la loi ne considère pas comme employés? \\ 1) Les personnes employées à titre de \\ (gérant, surintendant, contremaître, représentant de l'employeur dans ses relations avec ses employés ;}

Explication : D'après une résolution de la Commission des relations ouvrières en date du 16 juin 1944 le mot " contremaître " signifie " généralement la personne 
en charge d'un département ou de parties d'icelui, n'étant pas habituellement astreinte à un travail manuel et dont les fonctions et pouvoirs consistent soit à diriger la production, soit à ordonner et surveiller le travail de l'établissement, soit à engager ou congédier les employés ".

2) les directeurs et administrateurs d'une corporation ;

3) les professionnels ou ceux admis à l'étude des professions ;

4) les domestiques de maison ;

5) les personnes employées dans une exploitation agricole.

Explication : D'après la Loi du salaire minimum (S.R.Q., 1941, ch. 164), l'expression " exploitation agricole " signifie " une ferme mise en valeur par l'exploitant lui-même, ou par l'entremise d'employés ».

\section{VIII. - Quelles sont les procédures AVANT les
négociations?}

1) pour le syndicat ou l'association d'employeurs :

une requête écrite adressée à la Commission, accompagnée :

a) d'une résolution autorisant cette requête, dont une copie est attestée par la signature du président et du secrétaire ;

b) d'une copie ou exemplaire de sa constitution et de ses règlements ;

c) d'un état des droits $\left\{\begin{array}{l}\text { d'initiation, } \\ \text { d'entré, } \\ \text { de cotisation : }\end{array}\right.$

d) des noms et adresses des officiers et des représentants du syndicat.

$N . B .1)-$ Un ou plusieurs syndicats peuvent s'unir pour former la majorité absolue des employés.

2) Sur demande, la Commission fournit la formule nécessaire à la requête de reconnaissance.

2) pour la Commission:

a) elle procède à la vérification des livres et archives du syndicat ou de l'association d'employeurs.

Explication : c'est l'enquête des inspecteurs de la Commission

b) elle pourra reconnaître comme membre d'une association une personne qui remplira les conditions suivantes :

1) âge : au moins seize (16) ans ;

2) admission régulière comme membre et signature d'une carte d'adhésion dûment datée :

3) paiement personnel d'un droit d'entrée ou d'initiation d'au moins un dollar $(\$ 1)$;

4) cotisations : obligation de payer au moins cinquante cents (\$0.50) par mois ;

5) comme nouveau membre, paiement personnel des cotisations pour au moins un (1) mois ;

6) comme membre déjà initié : ne pas être en retard pour plus de trois (3) mois dans le paiement des cotisations ;

7) occuper régulièrement un emploi se rattachant aux occupations normales de l'employeur.

c) elle peut ordonner le vote au scrutin secret d'un groupe désigné d'employés, si

1) elle croit qu'une contrainte a été exercée sur certains d'entre eux pour les empêcher ou les forcer d'adhérer à un syndicat ;
2) les employés sont membres de plus d'un syndicat en nombre suffisant pour influer sur la décision.

Explication : Cela signifie que chacun des syndicats rivaux compte comme membre en règle au moins la majorité absolue des employés visés par les requêtes.

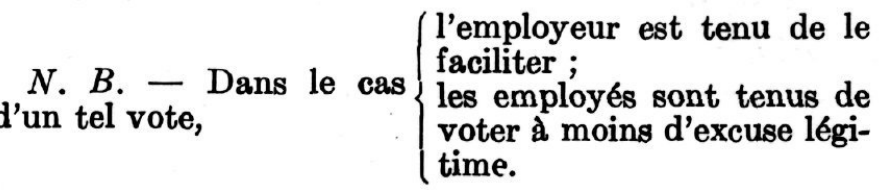

IX. - Quelle est la situation des parties durant la période comprise entre la date de la requête pour reconnaissance syndicale, et la fin des procédures pour la conclusion d'une convention collective?

1) les employés

ne peuvent faire une grève tant que :

a) leur syndicat n'a pas été reconnu comme représentant du groupe des employés en cause ;

b) toutes les procédures voulues pour la conclusion d'une convention collective de travail n'ont pas été épuisées ;

c) quatorze (14) jours ne se sont pas écoulés depuis la réception par le ministre du Travail dn rapport du conseil d'arbitrage sur le différend.

$N$. B. - Reste permise une interruption de travail qui ne constitue pas une grève.

2) l'employeur

ne peut

a) changer les conditions de travail de ses employés sans obtenir leur consentement ;

Explication : l'expression "conditions de travail " est prise dans son sens le plus général et signifie entre autres, les heures de travail, les salaires payés, les techniques de production, etc...

b) faire une contre-grève, c'est-à-dire refuser de fournir du travail à un groupe d'employés.

N. B. - Reste permise une interruption de travail qui ne constitue pas une contre-grève.

\section{X. - Quelles sont les conséquences d'une requête de reconnaissance?}

1) Pour la commission :

Dans le cas d'acceptation de la requête, émission d'un certificat de reconnaissance qui demeure la propriété de la Commission et qui, dans le cas de révocation, devra lui être retourné.

N. B. - La Commission avise par écrit l'employeur ou l'association d'employeurs dans le cas d'accord, refus, confirmation ou révocation d'un certificat de reconnaissance.

2) Pour l'employeur ou l'association d'employeurs : qui a reçu l'avis dont il est fait mention au (1) " de la question $\mathrm{XI}$,

$$
\text { obligation }\left\{\begin{array}{l}
\text { envers la totalité de ses employés } \\
\text { ou } \\
\text { envers les groupes déclarés distincts par la } \\
\text { Commission, }
\end{array}\right.
$$

a) de reconnaitre comme représentant collectif des employés ou des groupes d'employés à son emploi, les représentants du ou des syndicats groupant la majorité absolue des dits employés ou des dits groupes d'employés; 
Explication : les mots " majorité absolue " signifient cinquante pour cent $(50 \%)$ plus un employé. Exemple, s'il y a deux cents (200) employés, dans l'établissement ou dans le groupe, le syndicat doit en grouper au moins cent un (101).

b) de négocier de bonne foi, avec eux, une convention collective de travail.

Explication : l'expression " de bonne foi " signifie en vue d'en arriver à un accord.

3) Pour le syndicat:

qui a reçu l'avis dont il est fait mention à la note de (1) " de la question XI, obligation de négocier de bonne foi, avec l'association d'employeurs, une convention collective de travail.

\section{XI. - Quelles sont les procédures des NÉGOCIA- TIONS?}

1) Pour le syndicat reconnu:

il peut donner à l'employeur ou à l'association d'employeurs avec qui il veut négocier,

un avis écrit d'au moins huit (8) jours, indiquant la date, l'heure, et le lieu

où ses représentants seront prêts à le rencontrer luimême ou ses représentants ou ceux de l'association.

$N$. B. - L'association d'employeurs peut donner semblable avis au syndicat.

poursuivent sans suc-
cès pendant trente $(3)$

2) Chaque jours,

$\begin{array}{ll}\text { partie a le } & \text { b) une partie croit qu'il } \\ \text { droit si } & \text { b) }\end{array}$ ne sera pas possible de les compléter dans un délai raisonnable.

3) La Commission,

sur réception de tel avis, doit en informer le Ministre du Travail, lequel charge sans délai un conciliateur

a) de conférer avec les parties ;

b) de tenter d'effectuer une entente.

$N$. B. - Quel est le délai accordé au conciliateur?

Quatorze (14) jours, après quoi, il fait rapport au ministre; chacune des parties reste libre de consentir à une extension de ce délai.

\section{4) Le ministre du Travail}

doit, si le rapport du conciliateur atteste qu'une entente n'a pas été possible, nommer un conseil d'arbitrage suivant la Loi des différents ouvriers de Québec, (S.R.Q., 1941, ch. 167),

car le rapport du conciliateur tient lieu de la demande prévue en la dite loi.

\section{XII. - Quelle est la procédure APRÈS les négo- ciations?}

Chaque partie a le devoir d'en transmettre sans délai à la Commission, pour dépôt dans ses archives deux exemplaires ou deux copies certifiées (si la convention est reçue par un notaire).

La convention ne prend effet qu'à compter de ce dépôt.

$N$. B. - Le dépôt d'une convention conclue sous la Loi des syndicats professionnels se fait au bureau du ministre du travail et dispense les parties du dépôt à la commission, lequel est fait par le ministre.

\section{XIII. - Quelle peut être la durée de la convention collective de travail?}

Une convention peut être conclue pour une période maxima d'une (1) année.

On peut convenir qu'elle se renouvellera d'année en année, à moins qu'une des parties ne donne un avis écrit a l'autre partie,

dans un délai qui ne doit pas être de plus de soixante (60) ni de moins de trente (30) jours avant l'expiration de chaque période.

Explication : c'est un avis de non-renouvellement.

\section{XIV. - Quelle est la durée du certificat de recon- naissance?}

1) Pour le détenteur du certificat:

Une période d'un (1) an, renouvelable automatiquement, s'il n'y a pas opposition.

2) Pour le syndicat adverse:

Il peut, entre le soixantième (60e) et le trentième (30e) jour qui précèdent l'expiration ou la date de renouvellement de la convention collective,

présenter à la Commission une requête en vue d'être reconnu aux lieu et place du syndicat signataire, soit pour représenter $\left\{\begin{array}{l}\text { tous les employés } \\ \text { ou } \\ \text { un groupe mieux approprié. }\end{array}\right.$

\section{XV. - Quel est l'effet de la requête du syndicat' adverse si elle est acceptée par la Commis- sion?}

La convention collective de travail qui régissait les employés ou les groupes d'employés, pour lesquels le syndicat adverse vient d'être reconnu, cesse d'être en vigueur à partir de la date du renouvellement qui suit la date de la requête, nonobstant le défaut d'avis de non-renouvellement par l'une ou l'autre des parties.

\section{XVI. - Quelle est la situation des parties après la conclusion de la convention?}

(a) tant que le grief n'a pas été soumis à l'arbi-

1) a) Pour les employés :

toute grève est interdite

b) Pour l'employeur :

toute contre-grève est interdite trage en la manière prévue par la Loi des différends ouvriers de Québec; b) tant que quatorze (14) jours ne se sont écoulés depuis que la sentence a été rendue sans qu'elle ait été mise à effet.

N. B. - Reste cependant permise une interruption de travail qui ne constitue pas une grève ou une contregrève.

2) Le syndicat ou ses agents :

ne peuvent $\left\{\begin{array}{l}\text { ordonner } \\ \text { encourager } \\ \text { supporter }\end{array}\right\} \begin{aligned} & \text { un ralentissement d'activités } \\ & \text { destiné à limiter la production. }\end{aligned}$

3) Le syndicat minoritaire :

a) définition: s'il réunit au moins vingt (20) employés correspondant à au moins dix pour cent $(10 \%)$ du groupe soumis à la convention collective conclue par l'autre syndicat ;

b) ses droits: il peut soumettre par écrit au nom de ses membres, à l'employeur qui est partie à cette convention, tout grief résultant d'une violation de la loi ou de la convention collective de travail. 
4) l'employeur :

Lorsqu'un grief lui est soumis par écrit par le syndicat minoritaire, il doit immédiatement convoquer le représentant du syndicat qui est partie à cette convention et le représentant du syndicat minoritaire, pour être entendus lors de l'examen de ce grief.

XVII. - Quelle est la valeur d'une convention collective de travail qu'un employeur a conclue volontairement avec le syndicat représentant ses employés?

Explication : le mot " volontairement " signifie que le syndicat n'est pas reconnu par la Commission.

1) Cette convention est censée être conclue pour une période maxima d'une année et se renouveler automatiquement d'année en année ;

2) Cette convention demeure en vigueur

a) jusqu'au jour où un autre syndicat sera reconnu par la Commission pour représenter tous les employés qu'elle régit ou certains groupes d'entre eux ;

b) jusqu'à ce que la Commission décide, à la demande de l'employeur, qu'à la conclusion ou renouvellement de cette convention, le syndicat ne comptait pas parmi ses membres le pourcentage requis pour être reconnu suivant les dispositions de la loi.

\section{CHAPITRE DEUXIEME}

\section{ADMINISTRATION DE LA LOI}

Section A

\section{Pouvoirs de la Commision}

\section{XVIII. - Quels sont les pouvoirs de la Commis- sion?}

1) Elle a tous les pouvoirs de commissaires nommés en vertu de la Loi des Commissions d'enquête ; (S.R.Q., 1941, ch. 9).

2) elle peut faire des règlements pour régler

a) l'exercice de ses pouvoirs,

b) la procédure de ses enquêtes, etc ...

lesquels (approbation du lieutenant-gouverneur en entrent en Conseil

vigueur et

sur publication dans la Gazette officielle de Québec ;

3) s'il n'y a pas de règlement, elle peut dans toute affaire qui lui est soumise, prescrire tout acte ou formalité qui pourrait l'être par règlement et avec le même effet ;

4) elle peut autoriser des fonctionnaires à verifier en tout temps auprès de tout syndicat ou de tout employeur l'observance de la présente loi ;

5) elle peut émettre des certificats de reconnaissance ;

6) elle peut pour cáuse, reviser ou révoquer : a) toute décision et tout ordre rendus par elle ;

b) tout certificat qu'elle a émis.

Section B

Pénalités, sanction et poursuites prévues par la loi

XIX. - Quelles sont les pripcipales pratiques interdites pour lesquelles la loi prévoit des pénalités?

1) Pour un employeur ou une association d'employeurs ou un syndicat qui ferait défaut de se conformer au " 2) " ou au « 3) " de la question $\mathrm{X}$.

2) Pour quiconque qui con. (déclare ) une grève trairement aux dispositions provoque ou une . de la loi participe à contre-grève

3) Pour quiconque qui fait défaut de se conformer à une obligation ou à une prohibition imposée par la loi ou par un règlement ou une décision de la Commission.

4) Pour tout fonctionnaire de la Commission ou autre personne agissant. sous ses ordres, qui révèle à d'autres qu'à la Commission, un fait dont il a eu connaissance dans l'exercice de ses fonctions.

N. B. - 1) Une personne qui aide à commettre une infraction on conseille de la commettre, est partie à cette infraction est passible au même titre ;

dans le cas où l'infraction est commise par une corporation, une association d'employeurs ou un syndicat, est coupable de l'infraction tout directeur, tout administrateur, gérant ou officier qui, de quelque manière, approuve l'acte qui constitue l'infraction ou y acquiesce.

2) Si plusieurs personnes forment l'intention commune de commettre une infraction, chacune d'elles est coupable de chaque infraction commise par l'une d'elles dans la poursuite de la commune intention.

\section{XX. - La Commission peut-elle ordonner la disso- lution d'un syndicat ou d'une association d'employeurs?}

Oui,

a) lorsqu'un syndicat cherche à dominer ou à entraver la formation ou les activités d'une association d'employeurs ;

b) lorsqu'une association d'employeurs cherche à dominer ou à entraver la formation ou les activités d'un syndicat.

\section{XXI. - Comment s'intentent les poursuites pé- nales prévues par la loi?}

Une poursuite pénale ne peut être intentée

qu'avec $\left\{\begin{array}{l}\text { l'autorisation écrite de la Commission } \\ \text { le consentement du procureur général. }\end{array}\right.$

Marius Bergeron.

\section{NOTICES BIOGRAPHIQUES}

M arius Bergeron, L.L.D., L.s.soc., ancien élève de notre Faculté des Sciences sociales, actuellement attaché à la Confédération des Travailleurs catholiques du Canada comme conseiller technique.

EgBert Munzer, Docteur en droits civil et public, ancien membre du Ministère de l'Economigue du Reich Allemand ; plus tard professeur à l'Université de Saint-François-Xavier à Anti- gonish, et statisticien de l'université de Toronto; maintenant directeur du Département d'Économique à la Faculté des Sciences sociales de l'Université Laval.

Gérard Dion, L.Th., L.Ph., L.s.soc., professeur à la Faculté des Sciences sociales, secrétaire du Département des Relations industrielles et directeur de l'Office des recherches de ce département. 